\title{
Die „Deutsche Gesellschaft für Pneumologie und Beatmungsmedizin" feiert ihren 80. Geburtstag
}

\author{
The "Deutsche Gesellschaft für Pneumologie und Beatmungsmedizin" \\ Celebrates its 80th Birthday
}

Bibliografie

DOI $10.1055 / \mathrm{s}-2006-944252$

Pneumologie 2006; 60;

509-511

(c) Georg Thieme Verlag KG

Stuttgart · New York .

ISSN 0934-8387

Korrespondenzadresse

Prof. Dr. med. Nikolaus

Konietzko

Spillheide 78

45239 Essen

nikolaus.konietzko@t-online.de

\section{Hand aufs Herz}

Wer von uns kennt schon die Ursprünge unserer Gesellschaft? Für die meisten von uns, auch die Altvorderen, sind Ort, Zeit und nähere Umstände ihres Entstehens ein Mysterium, verborgen im Dunkel der Geschichte. Und offen gefragt, wen interessiert wirklich, wie alt die DGP, die Deutsche Gesellschaft für Pneumologie und Beatmungsmedizin ist, solange sie so prächtig wächst und gedeiht wie derzeit? Einen zumindest beschäftigt das Thema alle Jahre wieder, nämlich den jeweiligen DGP-Tagungspräsidenten. Spätestens dann, wenn er am Geleitwort zu „seiner“ Jahrestagung bastelt, beginnt er - exzellenter Pneumologe, aber unbedarfter Historiker - zu rechnen: Er zählt die vorangegangenen Kongresse zusammen, setzt die so gewonnene Zahl mit dem Alter der Gesellschaft gleich und teilt dieses einer staunenden Leserschaft mit. Aber leider ist seine Rechnung falsch. Sie lässt nämlich zwei Fakten außer Acht: Erstens, dass die Tagungen unserer Gesellschaft während der Kriegsjahre ausgesetzt wurden und zweitens, dass ab 1996 der Wechsel von einem zunächst zwei- auf einen einjährigen Rhythmus erfolgte.

\section{Wie alt ist unsere Gesellschaft denn also wirklich?}

Die Antwort findet sich in der Zeitschrift Beiträge zur Klinik der Tuberkulose (siehe Abb. $\bullet \mathbf{1}$ a-d). Den Hinweis darauf verdanke ich zwei Altpräsidenten, den Herren Werner Maaßen und Rainer Dierkesmann, die Kopien hat mir Herr Kropp aus seinem Tuberkulosearchiv besorgt. Die Zeitschrift Beiträge zur Klinik der Tuberkulose ist die Vorgängerin unserer heutigen Pneumologie. Im Vorwort zum 62. Band beschreibt der Chronist in blumigen Worten und mit einigem nationalem Pathos, wie sich im Mai 1926 in Danzig zwei der drei damals existierenden Tuberkulosegesellschaften, die Vereinigung der Lungenheilan- staltsärzte und die Gesellschaft deutscher Tuberkulosefürsorgeärzte zusammenschlossen, um die Deutsche Tuberkulosegesellschaft zu gründen. Die Deutsche Tuberkulosegesellschaft ist die Vorgängerin unserer heutigen Deutschen Gesellschaft für Pneumologie und Beatmungsmedizin. Interessant ist, dass die damalige dritte Kraft im Kampf gegen die Tuberkulose, das Deutsche Zentralkomitee zur Bekämpfung der Tuberkulose (DZK), erst Anfang der 90er-Jahre des vergangenen Jahrhunderts den engen Kontakt zur wissenschaftlichen Gesellschaft, der DPG, suchte und auch fand, was übrigens auch darin zum Ausdruck kommt, dass der gewählte Präsident der DGP qua Amt in Personalunion Präsident des DZK ist.

\section{Demnach ist der 24. Mai 1926 \\ der Geburtstag der Deutschen Gesellschaft für Pneumologie und Beatmungsmedizin.}

Die Gründung der Deutschen Tuberkulosegesellschaft erfolgte in politisch unruhigen und wirtschaftlich schwierigen Zeiten. Die junge Weimarer Republik litt damals schwer unter der Last der Reparationszahlungen an die Alliierten und unter der Hypothek der Versailler Verträge, an deren Folgen sie letztlich auch zu Grunde gehen sollte. Die Gründungsversammlung der Deutschen Tuberkulosegesellschaft fand in Danzig statt. Der Tagungsort Danzig war wohl nicht ohne Vorbedacht gewählt worden, man wollte „Flagge zeigen“. Danzig mit seiner überwiegend deutschsprachigen Bevölkerung war mit dem Vertrag von Versailles vom Deutschen Reich abgetrennt worden. Am 15. November 1920 war es zu einem unabhängigen Staat, der Freien Stadt Danzig, erklärt und unter die Aufsicht des Völkerbunds gestellt worden. „Reichsdeutsche“ konnten die Stadt nur über den polnischen Korridor errei- 
chen, wie im geschilderten Fall der 250 Tuberkuloseärzte, die mit einem Sonderzug aus Berlin angereist waren. Diese fraglos schwierige Situation sowie eine Vielzahl weiterer problematischer Verhältnisse gaben immer wieder Anlass für Beschwerden an den Völkerbund. Diese blieben aber zumeist unbeachtet, was wiederum unter der deutschsprachigen Bevölkerung Danzigs nationalistische Ressentiments förderte. Vor diesem historischen Hintergrund sind auch die für heutige Ohren stramm national klingenden Formulierungen in dem Bericht unserer Gründungsväter zu verstehen.

Heute ist Danzig eine polnische Stadt, die sich Gdansk nennt. Gdansk hat seine neue Rolle in der Brückenfunktion zwischen Ost und West in der Europäischen Union gefunden. Die Rolle von Gdansk ist damit nicht unähnlich der unserer Deutschen Gesellschaft für Pneumologie und Beatmungsmedizin. Die Einbindung der Kollegen aus dem Osten Europas in die pneumologische Gemeinschaft ist für die DGP eine Notwendigkeit, aus historischer Erfahrung gewonnen. Ein friedlich zusammenwachsendes Europa darf in Zukunft künstliche Inseln und widernatürliche Enklaven wie einst Danzig und Berlin oder heute Königsberg, durch verfehlte Politik entstanden, nicht mehr zulassen. Insofern erscheint mir die Beschäftigung mit der Geschichte unserer Deutschen Gesellschaft für Pneumologie und Beatmungsmedizin doch nicht ganz uninteressant, im besten Fall vielleicht sogar nützlich.

\section{BEITRÄGE ZUR KLINIK DER TUBERKULOSE} UND SPEZIFISCHEN TUBERKULOSE-FORSCHUNG

\author{
ORGAN DER DEUTSCHEN TUBERKULOSE-GESELISCHAFT \\ DER VEREINIGUNG DER LUNGENHEILANSTALTSÄRZTE \\ DER GESELLSCHAFT DEUTSCHER TUBERKULOSE-FÜRSORGEÄRZTE \\ UND DER
}

GESELLSCIIAFT PNEUMOTHORAX ARTEFICHALIS

UNTER MITWIRKUNG DER HERREN

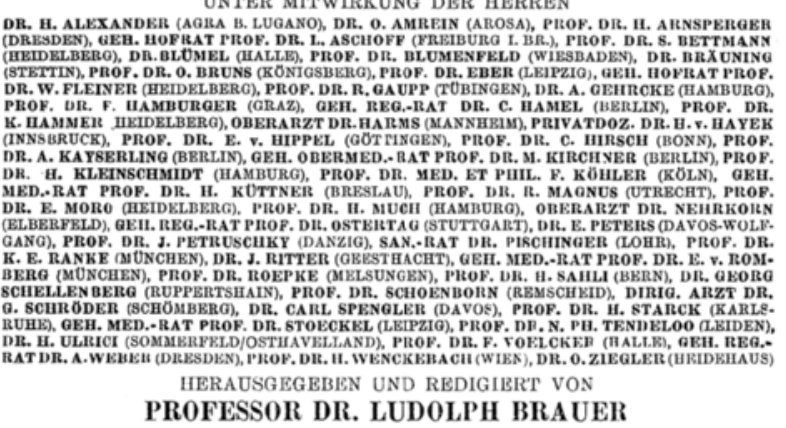

62. BAND

MIT 92 ABBHLUNGEN IM TEXT

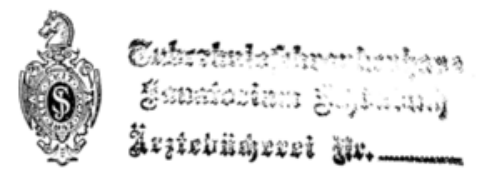

BERLIN

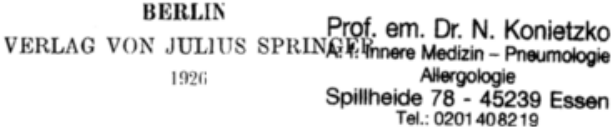

Vorwort.

Unsere alljährliche Tagung haben wir in diesem Jahre, einer Einladung des Senats des Freistaates Danzig durch Herrn Statsat Dr. Stade auf der vorjährigen Tagung in Koburg folgend, in der alten Stadt Danzig abgehalten, die durch den Versailler Vertrag gegen Recht und Billigkeit gezwungen aus dem deutschen Reich ausscheiden und als „Freistaat Danzig“ sich dem Völkerbunde unterstellen mußte, ohne bei diesem den Schutz zu finden, auf den sie vertragsmäßig Anspruch hat.

Die mannigfachen Schwierigkeiten, die durch die weite Entfernung Danzigs, besonders für die im Westen und Süden Dentschlands wohnenden Mitglieder, und für alle durch die rechtzeitige Beschaffung des polnischen Visums für die Fahrt durch den polnischen Korridor entstanden, waren nicht unerheblich. Durch die weite Entfernung Danzigs war es auch bedingt, daß alle Vorbereitungen nicht, wie sonst in der Regel, durch persönliche Rücksprache, sondern durch briefliche Verständigung geregelt werden mußten. Durch die großzügige und entgegenkommende Art, mit der die Vorbereitungen in Danzig, besonders durch Herrn Staatsrat Slade und durch den Vorsitzenden des Danziger Ärztevereins, Herrn Dr. Lohse, getroffen wurden, sind aber alle Schwicrigkeiten nicht zuletzt auch dureh die Unsicht, Erfahrung und Hingabe unseres Pischinger - überwunden worden. Kleine Unbequemlichkeiten der langen Hin- und Rückreise wurden von allen Teilnehmern gern in den Kauf genommen galt es doch, einem echt deutschen, gewaltsam von dem gemeinsamen Vaterlande abgespreng. ten Volksteil zu beweisen, daß auch deutsehe Ärzte und Wissenschaftler über alle künstlichen Schranken hinweg sich zı ihren Brüdern bekennen.

Alle Mühen und Unbequemlichkeiten wurden durch das, was uns in Danzig geboten wurde, überreichlich ausgeglichen. Das Beste aber war der deutsche Geist, der fest, still und klar alle unsere Veranstaltungen durehwehte und hi und wieder zu erhebendem und unvergeßliehem Ausbruch kam.

Am Freitag, den 22. Mai, tagte die Gencralversammlung des Deutschen Zentralkomitees zur Bekämpfung der Tuberkulose in Berlin. Durch ein Ent gegenkommen des Zentralkomitees, das seine Verhandlungen am Sonnabend, den 23., beschränkt hatte, um uns eine rechtzeitige Abfahrt zu ermöglichen, konnten simtliche Teilnchmer, wohl 250 an der Zahl, Berlin am Sonnabend, den 23. mit einem Sonderzug verlassen. Wenn auch die Fahrt durch den polnischen Korridor durch die polnische Revisionen für jeden Deutschen immer wieder ein innerlich schwer zu überwindendes Erlebnis ist, so kamen wir doch im wesentlichen unbehelligt am Abend zwischen 8 und $9 \mathrm{Uhr}$ in Danzig an, wo durch musterhafte Organisation rasch die bestimmten Unterkünfte gefunden und aufgesucht wurden. Die wissenschaftlichen Tagungen fanden in der Technischen Hochschule statt, in der uns bereitwilligst die $\Lambda$ ula mit vorzüglichem Licht. bilderapparat und gecigneten Nebenräumen für Geschäftssitzungen, für Berz-

Abb. 1 a -d Fotokopie aus dem 62. Band der Zeitschrift „Beiträge zur Klinik der Tuberkulose und spezifischen Tuberkuloseforschung“, der Vorgängerin der heutigen „Pneumologie“ (damals Springer-Verlag, heute Thieme-Verlag). Im Vorwort zum Verhandlungsbericht über eine gemeinsame Tagung von: „Der Vereinigung der Lungenheilanstaltsärzte“, „Der Gesellschaft Deutscher Tuberkulosefürsorgeärzte“ und „Der Gesellschaft Pneumothorax arteficialis “ vom 24. - 27. Mai 1926 in Danzig wird über die Gründung der „Deutschen Tuberkulose-Gesellschaft“, der Vorgängerin unserer heutigen DGP, berichtet. Die „Deutsche Tuberkulose-Gesellschaft“ ist demnach aus „Der Vereinigung der Lungenheilanstaltsärzte“ und „Gesellschaft Deutscher Tuberkulosefürsorgeärzte" hervorgegangen. 
tungen und andere Zweeke zur Verfügung gestellt war. Seiner Magnifizenz dem Herrn Rektor der Hochschule sei für sein Entgegenkommen der herzlichste Dank aller Teilnehmer ausgesprochen.

Die „Vereinigung " hielt schon am Sonntag, den 24. vorm. $8^{1 / 4}$ Uhr ihre erste Sitzung ab, an die sich sofort eine Geschäftssitzung anschloB. Montag, der 25. gehörte der "Gesellschaft deutscher Tuberkulosefürsorgeărzte", die am Vormittag ihre wissenschaftliche Sitzung abhielt, während die wissenschaftlichen Sitzungen der „Arbeitsgemeinschaft" am Dienstag, den 26. tagten. Der Vormittag des Mittwoch, den 27. war ursprünglich für wissenschaftliche Sitzungen der „Vereinigung" freigehalten; da aber zunăchst eine dringende und langdauernde Geschäftssitzung erforderlich wurde, mußte die wissenschaftliche Sitzung notgedrungen beschränkt werden, soda $\beta$ nur noch Steinmeyer mit seinem Vortrag über „Lungentuberkulose als Kriegsfolge“ zu Wort kam, während der Vortrag von Ritter „Die Diagnose und Prognose der Kavernen“ nicht mehr entgegengenommen werden konnte, da die Zeit zur Abfahrt nach Marienburg drängte.

Ober die nach unserer Meinung beachtenswerten Ergebnisse unserer wissenschaftlichen Tagung wird dieser Bericht der wissenschaftlichen Öfentlichkeit gegenüber Rechenschaft ablegen. Wir glauben annehmen zu dürfen, daß wiederum manche für die Behandlung, Bekämpfung und Erforschung der Tuberkulose wichtigen Ergebnisse gezeitigt sind.

Unvergeßlich werden daneben allen Teilnehmern die gesellschaftlichen Veranstaltungen bleiben, die uns in Danzig geboten wurden, in erster Linie der Empfang der Teilnehmer durch den Senat in dem altehrwürdigen Artushof mit der eindrucksvollen, von staatsmännischer und sozialer Einsicht und warmem Deutschtum getragenen Rede des Herrn Senatspräsidenten Sahm. Der daran sich anschließende Vortrag des Herrn Stadt-Archivar Dr. Recke über die Geschichte Danzigs hat es uns allen unvergeßlich eingeprägt, daß Danzig - entgegen der unwahren polnischen Propaganda - eine von Deutschen gegründete und auf deutschem Boden stehende Stadt ist, deren deutsche Gesinnung durch alle Schikanen Polens nicht unterdrückt, sondern gewachsen ist. Die leider vom Regen ein wenig getrübte Fahrt in die Danziger Bucht hinaus, der freundliche Empfang in Zoppot (mit seinen schőnen und verführerischen Spielsälen!), das Fest. essen im Gebäude der Loge ,Zur Einigkeit“ mit seinen ernsten und launigen Tischreden, die sachkundige und von Begeisterung getragene Rede des Herrn Oberbaurat Schmid und seine Führung durch die Marienburg - das stolze Wahrzeichen deutscher Kolonisations- und Verwaltungskunst, die gepaart war mit kriegerischer Tüchtigkeit - dazu das ernste, dabei künstlerisch entzückende Städtebild Danzigs, das in alle unsere Veranstaltungen hineinblickte. Wer wird sich nicht gern an diese erhebenden Stunden erinnern, die uns Reichsdeutschen immer wieder zum Bewußtsein brachten, wie schwer, wie treu und fest unsere deutschen Brüder in der östlichen Grenzmark um ihr Deutschtum ringen und kämpfen müssen, und wie in diesen Kämpfen das Bewußtsein ihres Deutschtums in ungeahnter Weise gewachsen ist. Neben diesen Veranstaltungen wurde für die Unterhaltung unserer Frauen durch sachkundige Führungen durch die Stadt mit ihren wunderbaren Kirchen und Sammlungen und durch die reizvolle Umgebung in dankenswerter Weise gesorgt. - Allen denen, die an dem Gelingen unserer
Tagung in so aufopfernder Weise mitgearbeitet haben, sei an dieser Stelle der herzlichste Dank des Vorstandes der Vereinigung und aller Teilnehmer ansgesprochen.

In den Geschäftssitzungen hat beide Vereine, die sich in einer ,Arbeitsgemeinschaft" zusammengefunden haben, die Frage in ausgiebiger Weise be schäftigt, ob in einer neu zu gründenden deutschen Tuberkulosegesellschaft alle wissenschaftlichen und ärztlichen Kräfte zur Bekämpfung, Erforschung und Behandlung der Tuberkulose zusammengefaßt werden sollten, oder ob die $3 \mathrm{Or}$ ganisationen, die sich dieser Aufgabe bisher gewidmet haben, das „Deutsche Zentralkomitee zur Bekämpfung der Tuberkulose “, die „Vereinigung der Lungenheilanstaltsärzte", die "Gesellschaft deutscher Tuberkulosefürsorgeărzte", durch gelegentliche oder dauernde Zusammenfassung ihrer Kräfte auf den alljährlichen Tagungen oder zu sonstigen Zwecken hinfort genügten. Die ,Vereinigung der Lungenheilanstaltsärzte" stellte sich fast einmütig auf den Standpunkt, daß es zweckmäßiger sei, alle ärztlichen und wissenschaftlichen Kräfte in einer großen Gesellschaft zusammenzufassen. Auch die Gesellschaft deutscher Tuberkulosefürsorgeärzte hat sich, trotz ernster und z. T. verständlicher Bedenken, schlieBlich auf diesen Standpunkt gestellt. Daß diese beiden Vereine, besonders die „Vereinigung“", die seit dem Jahre 1906 besteht, durch eine solche Neugründung sich selbst freiwillig in den Schatten stellt und sich mancherlei schöner wissenschaft. licher, kollegialer und gesellschaftlicher Rechte begibt, die ihr bisher zustanden, liegt auf der Hand. Aber wir waren alle der Uberzengung, daß es nicht auf die Belange einer einzelnen Gruppe ankommt - mögen sie noch so berechtigt sein sondern auf die rückhaltlose und tatkräftige Förderung der Bestrebungen, die der Allgemeinheit zunutze kommen sollen. So ist von den beiden Vereinen eine neue Gesellschaft, die „Deutsche Tuberkulosegesellschaft", gegründet worden. Ich verweise auf den am Schluß dieses Berichtes abgedruckten "Aufruf" und au die Satzungen und gebe namens unserer Vereinigung dem Wunsche Ausdruck, daß alle deutschen Ärzte und Wissenschaftler, die sich für den Kampf gegen die Tuberkulose einsetzen wollen, dieser Gesellschaft beitreten. Die beiden alten Vereine werden daneben nur noch ein bescheidenes Dasein zur Erledigung prak. tischer und kollegialer Fragen für den Heilstättenbetrieb führen und die Er. örterung großer wissenschaftlicher Fragen ihrem eigenen Kinde, dem sie eir rasches Wachstum wünschen, zuweisen. Die Vereinigung wird wie bisher besonderen Wert darauf legen, ihre Mitglieder freundschaftlich und kollegial zusammenzuführen und zusammenzuhalton. Daß dadurch auch wissenschaftliche und praktische Aufgaben in der Tuberkulosebekămpfung wesentlich gefördert werden, hat uns jede der zahlreichen Tagungen, die die Vereinigung bereits abgehalten hat, gelehrt.

Wir fordern alle Kollegen auf, in diesem Sinne treu zu ihrer alten Vereinigung zu halten, aber auch der neuen mit Vertrauen und dem ernsten Willen, der Bekämpfung der Volksseuche der Tuberkulose zu dienen, entgegenzukommen.

siehe Abb. 1 a 